\title{
Détecteur CTCSS pour relais radio embarqué dans un satellite amateur.
}

\author{
Thierry Capitaine,Valéry Bourny, Jérôme Dubois, Mohammed Hamzaoui, Aurélien Lorthois, Jacky Senlis \\ Etudiants : Marie DEVADASSAN et Ayoub BENMANSOUR \\ Jacky.senlis@u-picardie.fr
}

\author{
INSSET - Université de Picardie Jules Verne \\ 48 rue Raspail \\ 02100 ST QUENTIN
}

\begin{abstract}
RESUME : Depuis plusieurs années, avec mes collègues, nous avons mis en place une pédagogie de projets pour rendre nos étudiants actifs et responsables. Certains travaux ont déjà fait l'objet d'articles[1][2][3][4] présentés ces dernières années aux CETSIS. En 2011, notre établissement s'est impliqué dans un projet visant à concevoir l'intégralité des modules d'un picosatellite (CubeSat), avec l'objectif de le lancer en orbite en 2015/2016. C'est dans ce cadre que le projet «Conception et réalisation d'un détecteur CTCSS pour relais radio embarqué » a été proposé. Une étudiante de Master deuxième année et un étudiant de licence troisième année ont choisi ce sujet. Les masters ont alternativement une semaine de projet, puis une semaine de cours jusqu'à leur départ en stage et les étudiants de licence consacrent une journée tous les quinze jours au projet. L'intérêt de ce projet, hormis sa future utilisation dans un satellite amateur, est de permettre aux étudiants d'acquérir et de mettre en œuvre de façon concrète, leurs connaissances en traitement du signal et en électronique embarquée. En plus du travail scientifique et technique sur le sujet, les étudiants doivent mettre en place une gestion de projet, à l'image de ce qui se fait en bureau d'étude, avec des rapports et un suivi régulier des enseignants responsables.
\end{abstract}

Mots clés : dispositif pédagogique, électronique embarquée, traitement du signal, processeur de signal,, filtrage numérique, détecteur de tonalité.

\section{CAHIER DES CHARGES}

\subsection{Description}

CTCSS est l'acronyme de Continuous ToneCoded Squelch System, c'est un système utilisé couramment dans les transmissions radio, pour traiter uniquement les signaux audio qui comportent une tonalité subaudible particulière dans leur modulation.

Le sujet a été proposé par le président de l'AMSAT francophone, pour optimiser le fonctionnement d'un relais de communication radio destiné à être embarqué dans un satellite amateur (fig.1).

Le dispositif doit contrôler les communications audio à relayer par le satellite.

Pour économiser l'énergie, l'émetteur du relais ne doit fonctionner que si le signal audio reçu par le récepteur, contient une tonalité «subaudible» de fréquence paramétrable normalisée dans une gamme allant de $67 \mathrm{~Hz}$ à $250 \mathrm{~Hz}$.

Lorsque le signal audio contient la tonalité attendue, le relais doit passer en émission et réémettre le signal reçu, débarrassé de la tonalité (bourdonnement désagréable).

\subsection{Contraintes}

Dans le cahier des charges, figurent différentes contraintes sur les fonctionnalités à implanter et sur les caractéristiques électriques et thermiques du dispositif :

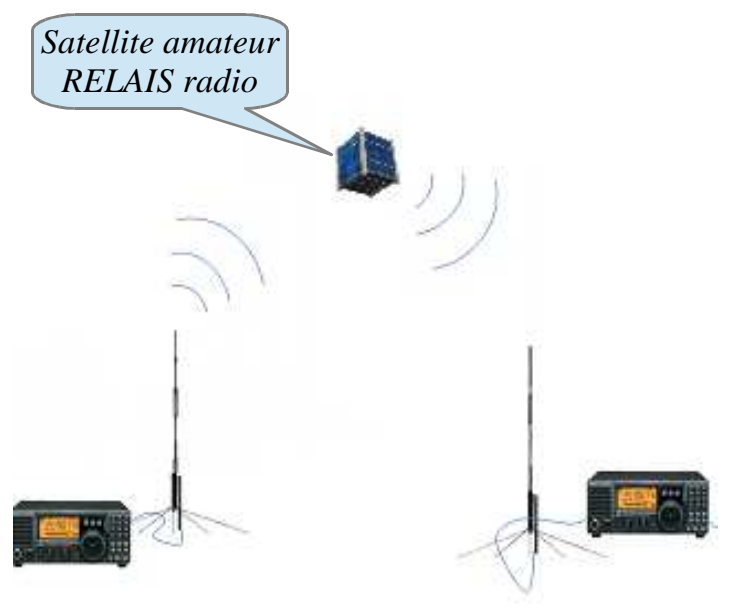

fig.1 Le satellite ne retransmet que les signaux contenant la tonalité subaudible

- Il doit être possible de choisir la fréquence de la tonalité à détecter par l'intermédiaire d'une liaison série.

- Le dispositif doit comporter une fonction pour désactiver le module.

- Le passage en émission sera commandé par une sortie logique quand il y a décodage de la tonalité attendue.

- La tension d'alimentation doit être égale à 3,3 volts ou à 5 volts.

- Le dispositif ne doit pas consommer plus de 4,5 $\mathrm{mA}$ lorsqu'il est alimenté en 5 volts. 
- La tolérance de détection de la tonalité doit être inférieure à $0,5 \%$

- Les caractéristiques du filtre réjecteur sont données :

- Atténuation minimale de la tonalité $30 \mathrm{~dB}$

- Ondulation : $<0.5 \mathrm{~dB}$

- Température de fonctionnement : $-40^{\circ} \mathrm{C} /+$ $70^{\circ} \mathrm{C}$

\section{DEROULEMENT}

\subsection{Recherche de solutions}

Les étudiants ont consacré la première semaine de projet à évaluer diverses solutions qui ont fait l'objet d' un rapport. Quatre solutions ont été envisagées et un tableau récapitulatif [6] fait par les étudiants regroupe les avantages, les inconvénients, la consommation et le prix de chacune d'elles. Deux des solutions sont écartées pour leur consommation excessive.

Une solution avec un composant dédié à cette application, de type MX465, a été écartée, car ce dernier ne dispose pas de communication série et nécessite donc l'adjonction d'un microcontrôleur. Cela conduit à une augmentation du prix et de la taille du dispositif.

Nous avons retenu la solution la moins chère qui est également celle qui consomme le moins d'énergie, elle est basée sur l'utilisation d'un processeur de signal.

\subsection{Description de la solution retenue (fig.2)}

Toutes les fonctionnalités décrites dans le cahier des charges vont être implantées de manière «soft », dans un seul composant électronique : un processeur de signal (D.S.P.). Ce processeur de signal doit posséder un certain nombre de périphériques :

- une entrée analogique pour la numérisation du signal audio reçu,

- une sortie à modulation de largeur d'impulsions (PWM) pour restituer le signal audio débarrassé de la tonalité subaudible,

- une entrée numérique pour désactiver le module,

- une sortie numérique pour faire passer en émission le poste émetteur, un port série pour le paramétrage de la tonalité à utiliser,

- une mémoire EEPROM pour stocker les informations de paramétrage,
- un oscillateur interne pour générer le signal d'horloge sans utiliser de quartz

- et enfin suffisamment de mémoire programme et de mémoire vive pour y implanter les algorithmes de détection et de filtrage.

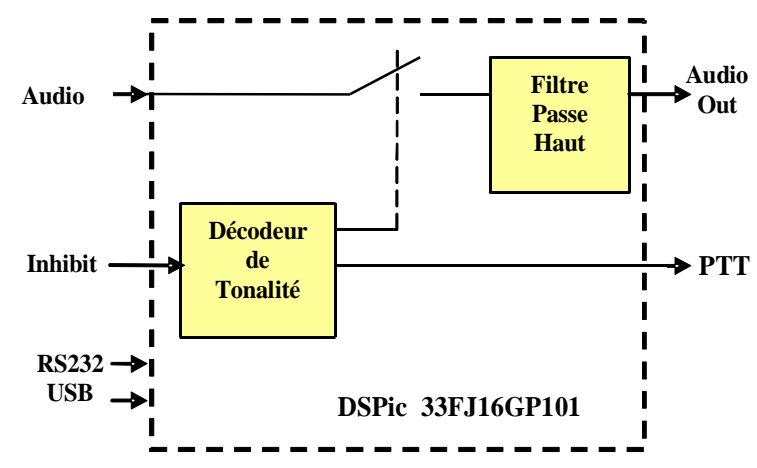

fig.2 Synoptique du module à réaliser

\subsection{Algorithme pour la détection CTCSS}

\section{2-3-1 Utilisation de la transformée de la TFD}

Nous nous sommes basés sur l'algorithme «détecteur de tonalité » qui a été décrit dans un article présenté au CETSIS en 2007 [5].

On utilise une forme discrétisée de la transformée de Fourier pour calculer l'amplitude de la composante fréquentielle $f_{0}$ recherchée (pulsation $\omega_{0}$ ).

L'astuce consiste à prendre une fréquence d'échantillonnage égale à quatre fois la fréquence du signal recherché soit $f e=4 f_{0}$ et à faire le calcul sur un nombre entier $N$ de périodes ( $4 . N$ échantillons). On obtient :

$$
\begin{aligned}
& \mathrm{F}\left(\omega_{0}\right)=\cdot \sum_{n=0}^{4 N-1} X(n T e) \cdot \exp \left(-j \omega_{0} n T e\right) \\
& =\sum_{n=0}^{\sum^{N-1} X(n T e) \cdot \exp \left(-j n \frac{\pi}{2}\right)} \\
& =[\underbrace{(X O-X 2+X 4-\ldots .)}_{X}-j \underbrace{(X 1-X 3+X 5-\ldots)}_{Y}]
\end{aligned}
$$

Le calcul de la transformée de Fourier discrète pour la tonalité recherchée, est alors très simple, il s'agit d'ajouter et soustraire alternativement les échantillons pairs pour obtenir la partie réelle $X$ de la transformée et impairs pour obtenir la partie imaginaire $Y$

On calcule ensuite l'amplitude $\boldsymbol{A}\left(\boldsymbol{\omega}_{0}\right)=\sqrt{X 2+Y 2}$

La détection est d'autant plus sélective que ce nombre de périodes est grand. Le signal audio peut être considéré comme un bruit non corrélé à la tonalité. 
Statistiquement il a autant de chance de s 'ajouter que de se soustraire à un échantillon, son influence sera d'autant plus faible que le nombre d'échantillons utilisés sera grand.

Le calcul est effectué sur un nombre entier $N$ de périodes du signal à détecter, en augmentant $\mathrm{N}$, on augmente la sélectivité mais également la durée du calcul.

\section{2-3-2 Tests de validation}

Les étudiants ont implanté l'algorithme de détection dans matlab et ont effectués des tests sur des signaux simulés puis sur des enregistrements réels provenant d'un poste radio doté d'un générateur CTCSS.

La figure 3 est un signal sur lequel les étudiants ont travaillé, l'analyse spectrale de la zone en rouge montre qu'il est possible de détecter la tonalité CTCSS dans une fenêtre de durée 100 millisecondes.

Le fichier matlab est téléchargeable à l'adresse [7]

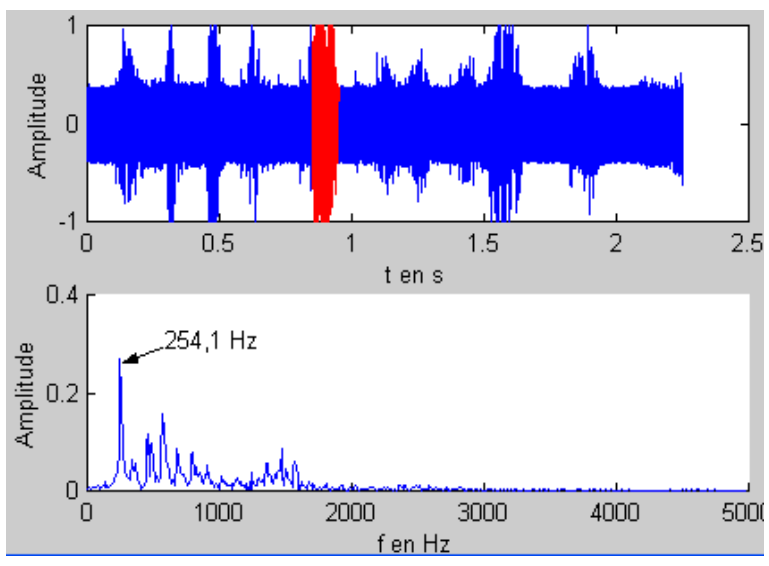

fig.3 Signal audio analysé sur une fenêtre de 100ms (en rouge) et le spectre de fréquence correspondant (fft)

Il est intéressant de comparer le résultat de notre algorithme au résultat obtenu par la f.f.t. traditionnelle, les étudiants ont été étonné de constater que le résultat de leur calcul était plus proche de l'amplitude recherchée que l'amplitude de la raie correspondante de la fft comme on peut le constater sur les copies d'écrans suivantes (fig.4 et fig.5).

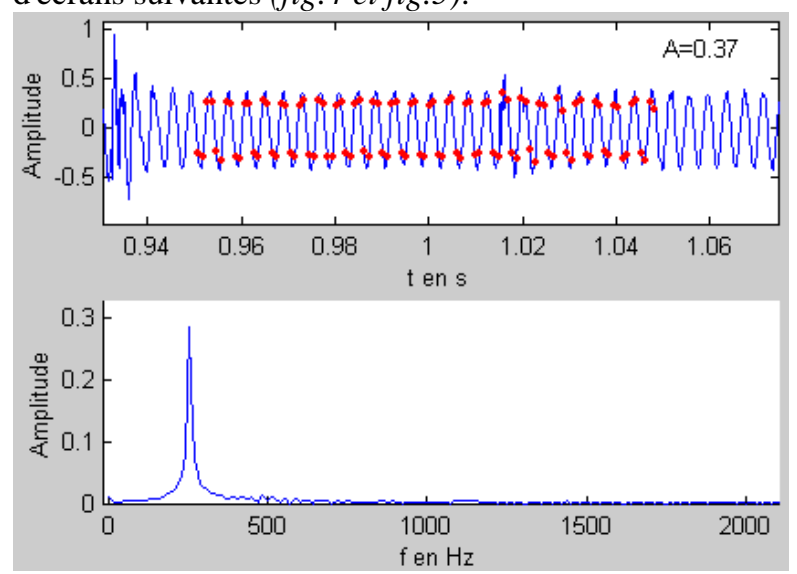

fig.4 Le calcul avec les points rouges donne $A=0,37$

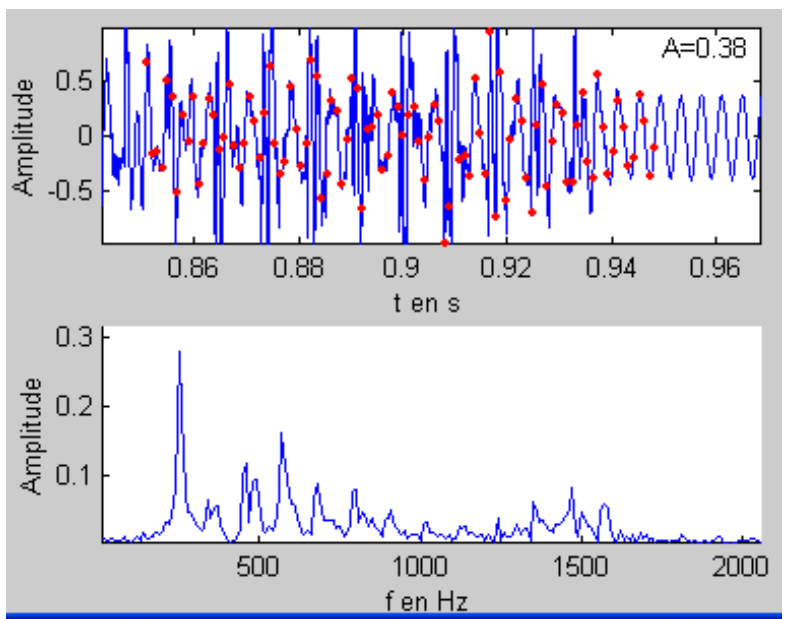

fig.5 On trouve $A=0,38$ malgré la voix

Ce travail a donné lieu à deux rapports [8] et [9]et a été validé en novembre.

\subsection{Algorithme du filtre réjecteur}

\section{2-4-1 Principe utilisé}

Pour débarrasser le signal audio de la tonalité CTCSS, on a choisi un filtre numérique à réponse linéaire finie (RIF) pour sa stabilité et ses performances facilement contrôlables.

Nos étudiants ont déjà mis en œuvre ce type de filtre dans un DSPic 30F6014 lors de travaux pratiques de traitement du signal [10], la réalisation du filtre réjecteur ne devrait pas leur poser de problème.

\section{2-4-2 Simulation et test du filtre}

L'algorithme du filtre réjecteur a été mis au point avec matlab et des enregistrements audio puis implanté dans le DSPIC d'une carte de TP.

La figure 6 montre un signal avec une tonalité CTCSS avant et après filtrage.
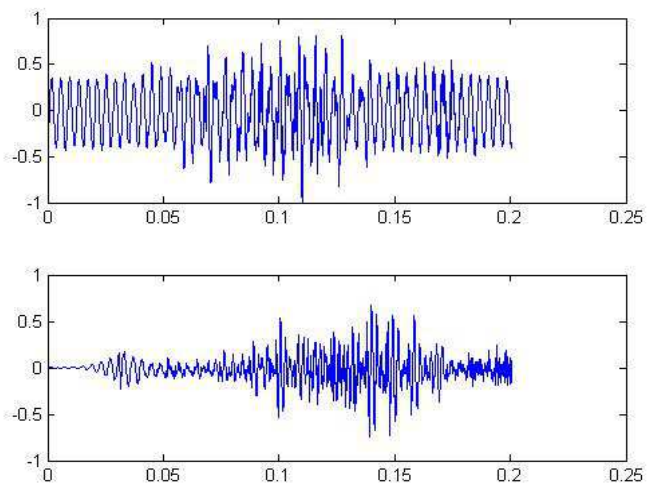

fig.6 Filtrage du signal audio

Ce travail a été présenté fin décembre lors d'une soutenance orale [11] par l'étudiante de M2 devant un jury d'enseignants 


\subsection{Test sur carte d'évaluation}

L'implantation des algorithmes dans une carte d 'évaluation basée sur un DSP est en cours. Une fois cette étape validée, les étudiants pourront passer à la phase de prototypage du dispositif .

\subsection{Collaboration entre étudiants de L3 et de M2}

Le DSP de la carte d'évaluation n'ayant pas toutes les performances requises par le cahier des charges, il faut concevoir et réaliser une carte électronique pour tester le DSP qui a été choisi. Ce travail a été confié à l'étudiant de L3 qui le réalise avec le logiciel de C.A.O. électronique «PROTEUS » en suivant les conseils de l'étudiante de M2.

L'étudiant vient de terminer la phase d'apprentissage du logiciel et commence la réalisation de la carte électronique.

\subsection{Tests et validation du prototype}

La dernière phase du projet consistera pour les étudiants à vérifier le bon fonctionnement du dispositif et surtout sa conformité au cahier des charges.

\subsection{Dossier technique, rapport et soutenance}

Le projet se termine par une présentation orale de quinze minutes, suivie de questions, devant un jury d'enseignants, en présence des étudiants de la formation Les étudiants doivent également rendre un mémoire avec un dossier technique complet sur leur réalisation.

\section{CONCLUSION}

Le fait d'alterner semaines de cours et semaines de projets permet aux étudiants en master d'être très efficaces dans l'avancement de leur projet. Cela nécessite en contrepartie une organisation spécifique : il faut permettre aux étudiants d'accéder au laboratoire et à la salle projet toute la semaine (de $8 \mathrm{~h} 30$ à $17 \mathrm{~h}$ ), contrôler leur assiduité. La présence et la disponibilité d'enseignants et de personnel de laboratoire est indispensable. Cela est relativement facile à implanter sur de petites structures comme la nôtre ( 30 étudiants sur l'ensemble du parcours systèmes embarqués). Cette organisation a été mise en place en 2011, en même temps que l'ouverture de notre Master à l'alternance.

Une manipulation avec le prototype développé par les étudiants est prévue au colloque 2013.

\section{Bibliographie}

[1] Thierry CAPITAINE - Valéry BOURNY - Ludovic BARRANDON - Jacky SENLIS

Étudiants : Didier CHAMPAGNE - Cyril DROMAS Julien DAUTREPPE.

Transmission d'images sur canal audio à faible bande passante: Application aux technologies spatiales. (CETSIS 2011)

[2] Valéry BOURNY - Thierry CAPITAINE - Florent SWINGEDOUW - Aurélien LORTHOIS - Jacky SENLIS - Baptiste DOS SANTOS.

Procédure d'étalonnage des outils et des pièces dans les procédés d'usinage. (CETSIS 2011)

[3] Tierry CAPITAINE, Valéry BOURNY, Mohammed HAMZAOUI,Aurélien LORTHOIS, Ludovic BARANDON, Jacky SENLIS

Décodage des trames émises par les balises de détresse 406.025 MHz (CETSIS 2009)

[4]Thierry CAPITAINE, Valery BOURNY, Mohammed HAMZAOUI, Aurelien LORTHOIS, Jacky SENLIS

Démodulation et décodage de trames AX25 par DSPIC pour la localisation d'un ballon sonde météo dans le cadre d'une action "Planète Sciences »(CETSIS 2008)

[5]Thierry CAPITAINE, Valery BOURNY, Pascal CARTON, Mohammed HAMZAOUI, Jacky SENLIS Implantation d'un algorithme de traitement du signal sur cible à faibles ressources: Identification de chaînes DTMF avec un microcontrôleur en boîtier DIL8 (CETSIS 2007),

Journal sur l'enseignement des sciences et technologies de l'information et des systèmes 2008, J3eA 7 HORS SÉRIE 1 (2008) page 1027

[6] http://jgsenlis.free.fr/ctcss/tableau.pdf Tableau sur le choix du microcontrôleur fait par les étudiants

[7] http://igsenlis.free.fr/ctcss/ctcss.zip

Script matlab et enregistrement pour les tests

[8] http://jgsenlis.free.fr/ctcss/Rapport_Sem39.pdf

Rapport des étudiants de la semaine 39

[9] http://jgsenlis.free.fr/ctcss/Rapport_Sem43.pdf

Rapport des étudiants de la semaine 43

[10] http://jgsenlis.free.fr/dspic M1.htm

Filtre RIF sur DSPic (cours et Travaux Pratiques)

[11] http://jgsenlis.free.fr/ctcss/soutenance_CTCSS.pdf Diaporama de la soutenance orale de Melle Marie Devadasan 